\title{
FUSELAGE STRUCTURE RESPONSE TO BOUNDARY LAYER, TONAL SOUND, AND JET NOISE
}

\author{
L. Maestrello* \\ NASA Langley Research Center \\ Hampton, VA 23681
}

\begin{abstract}
Experiments have been conducted to study the response of curved aluminum and graphite-epoxy fuselage structures to flow and sound loads from turbulent boundary layer, tonal sound, and jet noise. Both structures were the same size. The aluminum structure was reinforced with tear stoppers, while the graphite-epoxy structure was not. The graphite-epoxy structure weighed half as much as the aluminum structure. Spatiotemporal intermittence and chaotic behavior of the structural response was observed, as jet noise and tonal sound interacted with the turbulent boundary layer. The fundamental tone distributed energy to other components via wave interaction with the turbulent boundary layer. The added broadband sound from the jet, with or without a shock, influenced the responses over a wider range of frequencies. Instantaneous spatial correlation indicates small localized spatiotemporal regions of convected waves, while uncorrelated patterns dominate the larger portion of the space. By modifying the geometry of the tear stoppers between panels and frame, the transmitted and reflected waves of the aluminum panels were significantly reduced. The response level of the graphite-epoxy structure was higher, but the noise transmitted was nearly equal to that of the aluminum structure. The fundamental shock mode is between $80^{\circ}$ and $150^{\circ}$ and the first harmonic is between $20^{\circ}$ and $80^{\circ}$, for the underexpanded supersonic jet impinging on the turbulent boundary layer influencing the structural response. The response of the graphite-epoxy structure due to the fundamental mode of the shock impingement was stabilized by an externally fixed oscillator.
\end{abstract}

\section{Introduction}

The anechoic wind tunnel at Langley can accommodate a section of curved full-scale fuselage structure. Experiments were conducted to investigate the response and assess the use of graphite-epoxy structures to replace aluminum ones. Load, response, and noise radiated from two structures forced by a turbulent boundary layer, tonal sound, and jet noise were investigated. The methods adopted were general and apply to analysis of and observations from the instability of flow, structure, and sound radiation. The goal was to extract physical information from experiments for modeling and developing prediction methods applicable to nonlinear convecting fluid and sound load problems. A classical problem in structural dynamics is the response and transmission of sound

\footnotetext{
"Senior Research Scientist, Associate Fellow
}

through a flat plate. However, aircraft structures are curved, pressurized, and forced by a turbulent boundary layer and the sound from the power plant. Little is known about how these features affect the response and noise transmission on curved structures. For these reasons, experiments and numerical simulations have been conducted to obtain results useful for industrial applications. ${ }^{1-3}$

The wall pressure fluctuation generated by the turbulent boundary layer is nonlinear and so is the response of the structure when a high intensity sound is imposed. The theory of boundary layer instability was discussed comprehensively in Landau and Liftshitz. ${ }^{4}$ In the study presented here, the spectrum distribution contained harmonic peaks on the broadband and the dynamics changed over a wide range in the phase space. Depending on the levels of the tonal sound and jet noise, the structure manifested different types of responses, from periodic to spatiotemporally chaotic. A systematic analysis of the data were done to detect aperiodic waves and the breakdown to chaotic state response. $^{\text {5-7 }}$

A number of papers has been written on nonlinear processes of an elastic panel, such as nonlinear modal interaction and chaotic behaviors. ${ }^{8-10}$ To seek an understanding of the present experimental data, 11,12 mathematical models were introduced ${ }^{1-3}$ to study the stability of the structure and to demonstrate the feasibility of active control. Recently, several significant contributors have studied the general and specific aspects of temporal and spatiotemporal chaos, including control of chaotic responses in spatially developing flows. ${ }^{13-19}$

Experiments on the excitation and control of a frame-stringer structure forced by shock noise from a jet exhaust were reported. ${ }^{20}$ Early experiments recognized that the instability of the jet column, due to the feedback loop between fluid flow and sound, was the cause of a powerful acoustic tone. ${ }^{21}$ Later work shows that the self-excited oscillation (screech tone) contributes to the sound amplification at angles of $30^{\circ}$, $90^{\circ}$, and $120^{\circ}$ from the jet axis.22,23 Numerical work on the panel response forced by the sound of subsonic and supersonic jets demonstrates that the acoustic load is a result of instability. ${ }^{24}$

The present paper examines three areas of interest, one is the nonlinear responses of aluminum and graphite-epoxy structures, the second is the control of the responses and the third is the transmission of acoustic energy. The acoustic wavelengths are long enough, so that the spatial growth of the fundamental mode and harmonic mode are significant as the instability of the boundary layer grows. 
This paper is organized as follows. Section 2 describes the test facility and the unique anechoic test section, the geometry of the models, and instrumentation. Section 3 is concerned with the data analysis methods for nonlinear system response. Section 4 presents the main results of the study. Section 5 describes the wave transmitted through the structures. Section 6 describes the control of structure response. Section 7 is the conclusion.

\section{Test Facility, Models, and Instrumentation}

Experiments were conducted in a subsonic wind tunnel with an anechoic test section to study boundary layer and sound interaction problems in a simulation of an airplane fuselage structure in a flight environment (fig. 1(a)). In these experiments, the structures were curved fuselage types, one of aluminum (fig. 1(b)) and the other of graphite-epoxy (fig. 1(c)). The aluminum panel was approximately twice the weight of the graphite-epoxy. The structures were mounted on the side wall of the test section opposite to the anechoic wall where the acoustic source was located. The structures were $0.609 \mathrm{~m}$ wide, $3.048 \mathrm{~m}$ long in the direction of flow, with a radius of curvature of $2.529 \mathrm{~m}$. The aluminum structure was machined from a plate into six panels separated by one longitudinal and two lateral tear stoppers equally spaced at distance L (figs. 1(a) (b)). The panels were $0.0109 \mathrm{~cm}$ thick and the

(a)

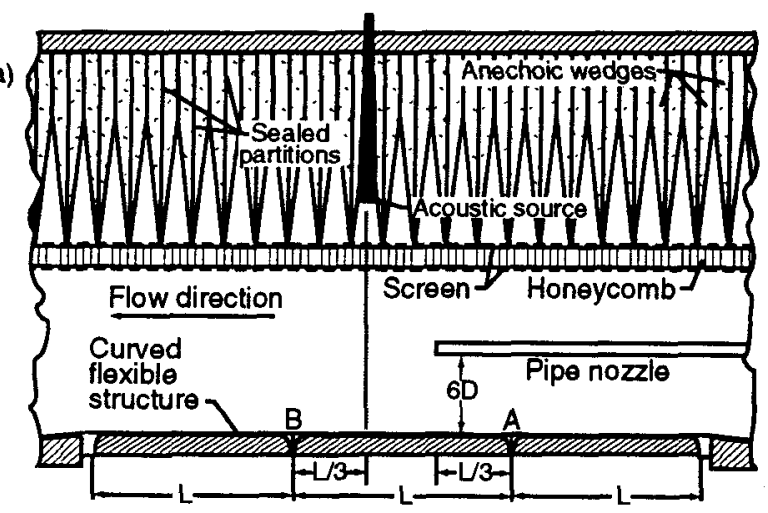

(b)

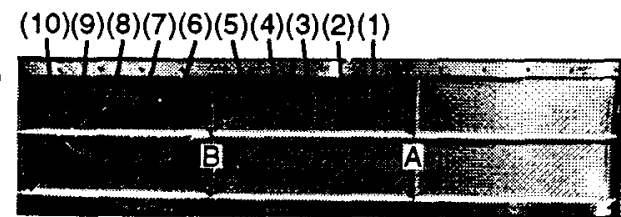

(c)

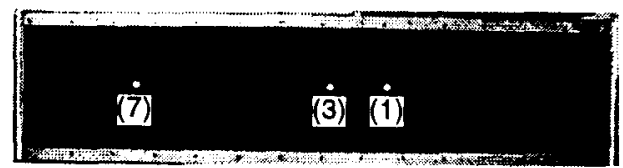

Fig. 1. Wind tunnel test section: a) anechoic section, b) view' of the aluminum structure, c) view of the graphite-epoxy structure. boundaries between panels and tear stoppers and the outer frame were smooth with 1.25 -in-radius of curvature to minimize the amplitude of reflected waves at the boundaries. The geometry of the tear stoppers was a departure from the standard blunt discontinuity used in aircrafts. Past experiments using soft boundaries (rubber wedges) or a gradual increase in the metal thickness toward the rigid frame indicate significant reduction in response and interior noise in wind tunnel and in flight experiments. 25,26 The graphite-epoxy structure was $0.021 \mathrm{~cm}$ thick and was made of seven layers oriented at $45^{\circ}, 90^{\circ}$, and $-45^{\circ}$ in a single curved panel without tear stoppers. The graphiteepoxy structure was designed to eliminate the surface discontinuity and the sound due to wave scattering at the boundaries (fig. 1(c)) .

The wind tunnel anechoic side wall is a unique construction that is designed to minimize broadband acoustic pressure at the test section. It consists of low frequency absorbers, which are $1 \mathrm{~m}$ long anechoic wedges and a high frequency absorber, which is a $0.125 \mathrm{~m}$ thick honeycomb panel with fine screens at the surfaces. Each wedge partition is air-sealed from the others to prevent outflow and inflow across the screens due to pressure gradient along the flow direction. The acoustic sources are created by four 120-watt phaseamplitude matched speakers mounted on a diffuser within the anechoic side wall facing the structure and centered at L/3 downstream of the second tear stopper (figs. 1(a), (b)). The frequency of the sound was set to $1225 \mathrm{~Hz}$ at the power level of $134 \mathrm{~dB}$ (re. $10^{-12} \mathrm{~W}$ ). The nozzle consists of a long pipe with a diameter of $0.0508 \mathrm{~m}$ placed at the center of the test section at $\mathrm{L} / 3$ downstream of the first tear stopper (figs. 1(a), (b)), where $L=1.016 \mathrm{~m}$ or $1 / 3$ of the length of the structure. A pipe nozzle is used because of the reduced outer flow wake profile at the nozzle exit when compared with a convergent nozzle.

The Reynolds number per meter Re/m of the turbulent boundary layer was $2.95 \times 10^{5}$, the free stream velocity Ue was $52 \mathrm{~m} / \mathrm{sec}$, and boundary layer thickness was $0.058 \mathrm{~m}$. The mean velocity profile was measured with hot wires. The wall pressure fluctuation was measured with miniature pressure transducers mounted flush with the inner surface and protruding the tear stoppers between the panels. The number of spatial locations at which the wall pressure could be measured was limited. As a result, the spatial extent was difficult to ascertain. Figures 1(a), (b) show location $\mathrm{A}$ at $-6.8 \mathrm{D}$ or $131.4^{\circ}$, and location $\mathrm{B}$ or $23.8^{\circ}$ from the jet axis. Location A contains a single pressure transducer and location B contains one longitudinal and one lateral transducer array. The pressure transmitted through the panel to the outside was also measured by a pressure transducer located $1.5 \mathrm{~m}$ from the panel. The vibration response was measured by miniature accelerometers. The accelerometers (1 to 10, fig. 1(b)) were equally spaced between tear stoppers at $5.08 \mathrm{~cm}$ intervals on the aluminum structure. For the graphite-epoxy structure, two accelerometers were located at 1,3 , and 7 
(fig. 1(b), (c)). All measurements were made from dc current response.

\section{Data Analysis}

Several difficulties existed in the interpretation of the experimental results, in the classification of the responses, and in the selection of the observed data. In the laboratory, waves in flows and on structures evolve in space and in time. Noise from a jet is produced by both axisymmetric and helical modes. High amplitude screech tones from the jet and tonal harmonics from the acoustic sources are observed in combination with broadband spectrum, each having different directivity and power level. ${ }^{22}$ Extracting physical information from experiments is essential to understanding the dynamics of unsteady loads. The information needed includes

(a) data relevant to tonal sound coupling with the boundary layer

(b) the effects of the screech mode direction and level on broadband response

(c) local and global dynamics of acoustic radiation

(d) rescaling data into full size engine noise loads on a fuselage structure in forward motion

The time trace of the measured quantities, which is irregular, quasi-periodic, or chaotic, was used for the interpretation of the experimental observations. The time history of the wall pressure fluctuation, structure acceleration response, and acoustic transmitted pressure were measured. The power spectrum density, the phase portrait, the probability distribution, and the Liapunov exponent are evaluated from the time history. ${ }^{12}$ For a nonstationary signal $q(t, x)$, such as the pressure fluctuation $p(t, x)$ or panel acceleration $g(t, x)$, the instantaneous power spectrum at instant $T$ is defined by

$$
P(f, T)=\left|\frac{1}{2 \pi} \int_{T-I / 2}^{T+I / 2} \exp (i 2 \pi f t) p(t, x) d t\right|^{2}
$$

where $T$ is chosen so that the experimental run contains the interval $T-(I / 2), T+(I / 2)$ for a sufficiently large $I$. The probability density of $q(t, x)$ is denoted by $Q(r, t)$.

The Liapunov exponent of the panel motion at point $L(x, y)$ is given approximately as

$$
\lambda(E, L)=\frac{1}{E} \ln \left\{\frac{|z(E, x, y)|}{\left[\int_{0}^{E} z^{2}(t, x, y) d t\right]^{1 / 2}}\right\}
$$

For a duration $E$ of run, where $z(t, x, y)$ denotes the panel displacement at time $t$ and point $L$. Given the measured acceleration $g(t, x, y)$, the displacement $z$ can be computed by two-term Taylor series expansion for a small time increment of $\Delta t$
$z(t+\Delta t, x, y) \approx z(t, x, y)+\dot{z}(t, x, y) \Delta t+\frac{1}{2} g(t, x, y)(\Delta t)^{2}$

where

$$
\dot{z}(t, x, y)=\frac{\partial}{\partial t} z(t, x, y) \approx \frac{1}{\Delta t}[z(t, x, y)-z(t-\Delta t, x, y)]
$$

Let $t_{0}=0, t_{i}=i \Delta t, i=1,2, \ldots, N$ with $t_{N}=E$. Choose the initial conditions $z(-\Delta t, x, y)=z_{-1}$ and $z(0, x, y)=z_{0}$ properly or arbitrarily. If $\lambda$ is independent of the initial data, the above formula yields

$$
z\left(t_{i+1}, x, y\right) \approx 2 z\left(t_{i}, x, y\right)-z\left(t_{i-1}, x, y\right)+\frac{1}{2} g\left(t_{i}, x, y\right)
$$

and the integral can be evaluated by the finite sum

$$
\int_{0}^{E} z^{2}(t, x, y) d t \approx \sum_{i=1}^{N} z^{2}\left(t_{i}, x, y\right) \Delta t
$$

The Liapunov exponent $\lambda(E, x, y)$ determines the local stability of the periodic panel motion; stable if $\lambda<0$ and unstable if $\lambda>0$. But it may change sign as point $L$ varies. It is known that for $\lambda>0$ the motion, depending on sensitivity to the initial conditions, may become chaotic. $^{27}$

Two of the steps should be the estimation and validation of an adequate model representing the physical system and comparing a set of measurements. These procedures will suffer from a lack of consistency when the model being validated is sensitive to initial conditions. ${ }^{28-30}$ Computer based methods for the analysis of time signals have become an important tool for characterizing the dynamics. Coupling between spatial and temporal fluctuation has been shown using the time series of a system showing high-dimensional spatiotemporal chaos. 31,33 In the laboratory, no measurement can be performed with extreme accuracy and therefore no matter how good an initially identified model is, the predictions will worsen in the course of time. Consequently, comparison will not be of much help in validating identified models if they are sensitive to initial conditions since the quality of predictions is proportional to the accuracy with which the initial state was measured.

\section{Experimental Results}

Pattern selection of the wall pressure fluctuations was observed. This selection occurs intermittently. The most complex dynamic response is not governed by the turbulent boundary layer alone, but by the turbulent boundary layer in the presence of high intensity tonal sound and sound from high subsonic jet or from an underexpanded supersonic jet. As nonlinearity increases with the increase in the sound level, the load and the response assume deterministic spatiotemporal distributions over the surfaces of the structure, and eventually lead to chaotic motions. $7,18,19$ 


\section{4a. Wall Pressure Eluctuation Without and With Acoustic Forcing}

The turbulent boundary layer in the wind tunnel is receptive to high intensity sound; as a result, the thickness and the wall pressure fluctuations increase. ${ }^{30}$ The measured real time pressure $p(t)$, the computed power spectral density $P(f, T)$, the phase plots of the computed $\dot{p}(t)$ versus $p(t)$, and the computed probability density $Q(r, T)$ are shown in figures 2(a), (b), (c), (d). These figures show

(a) turbulent boundary layer at $13.6 \mathrm{D}$

(b) turbulent boundary layer with pure tone sound at $13.6 \mathrm{D}$

(c) turbulent boundary layer and sound from underexpanded jet at $13.6 \mathrm{D}$

(d) turbulent boundary layer with sound from an underexpanded jet at $-6.8 \mathrm{D}$

The real time pressure $p(t)$ is shown for an interval of $0.02 \mathrm{sec}$ near the instant $T$ and was used for the evaluation of the instantaneous plots of spectrum, phase, and probability. The spectrum of the pressure fluctuations, varying from 40 to $140 \mathrm{~dB}$, is also shown.
Figure 2(a) shows the wall pressure fluctuations of the turbulent boundary layer with the broadband spectrum, convective phase portrait, and nearly Gaussian distribution. In figure 2(b) the effects of pure tonal sound show peaks of $30 \mathrm{~dB}$ above the broadband with two harmonics of $2 \mathrm{f}$ and $3 \mathrm{f}$. The time plot is temporally chaotic with virtually no periodicity behavior. The phase is skewed, which indicates convection and rotation. The probability from the turbulent boundary layer alone has a quasi-zero mean and is nearly symmetric, whereas the plot with the superimposed tonal sound is clearly non-Gaussian and has a much larger standard deviation than that in figure 2(a). This difference in broadband spectrum shows that the distribution of energy in the boundary layer is altered by the incident tonal sound as dramatically shown in a previous experiment where the tone was at a lower frequency. Figures 2(c), (d) show the wall pressure fluctuations and sound from the underexpanded jet at two locations. The bandwidth increases from $10 \mathrm{kHz}$ to $20 \mathrm{kHz}$ since the noise from the jet is distributed over a broader frequency range. Screech modes are found to be present at operating conditions. The fundamental screech tone is detected upstream of the nozzle at location A (fig. 1). The first (d)

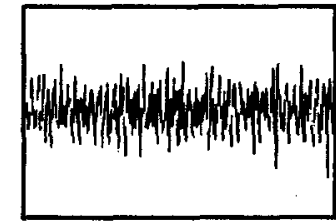

(c)

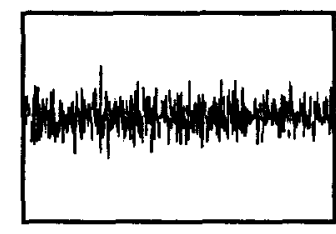

(b)
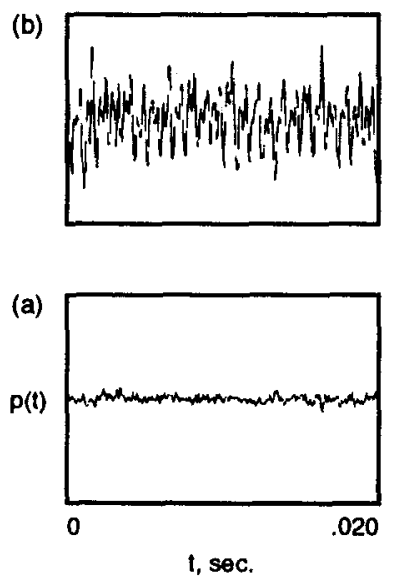
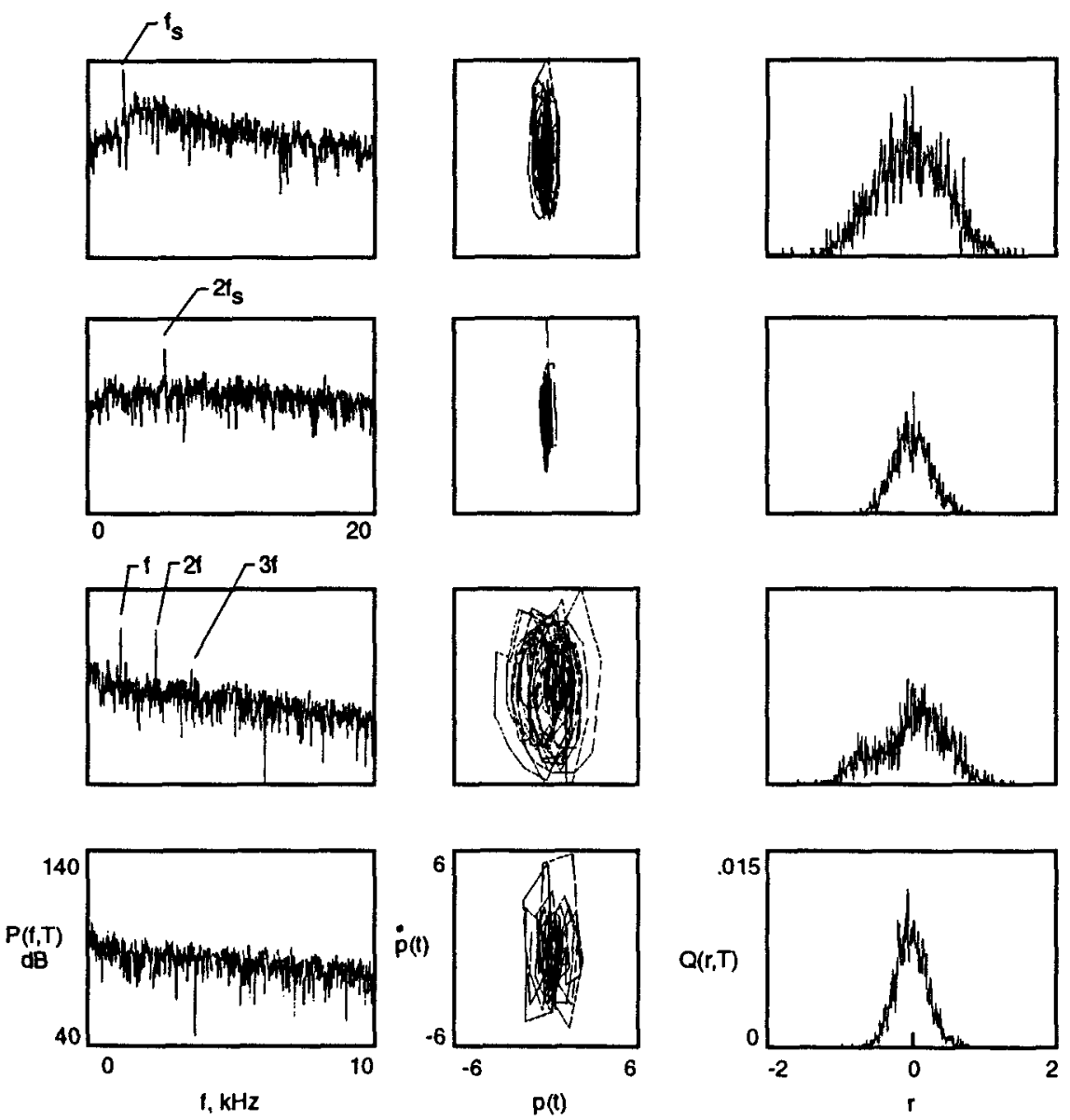

Fig. 2. Wall pressure fluctuations. 
harmonic is oriented downstream of the nozzle at about $23.8^{\circ}$, location B. Random angular peak amplitude variations are noticeable in both fundamental and harmonic. The results are qualitatively similar to that of previous experiments. ${ }^{21,27}$ The amplitude of the broadband spectrum becomes nearly constant (figs. 2(c), (d)), while in figures 2(a), (b) it is higher at the low frequency end due to different loads. The real time pressure fluctuation in figures $2(\mathrm{c})$, (d) consists mainly of $\mathrm{N}$-waves of random amplitude. In the spectrum plots, the peaks of the fundamental and harmonic are nearly $30 \mathrm{~dB}$ above the broadband. In the phase portrait, the convective effect is observable and the probability plots are nearly Gaussian.

\section{4b. Structural Response}

The static pressure inside the wind tunnel was below the ambient pressure outside the wind tunnel in simulation of an aircraft structure load in flight. Thus, the structure in the tunnel tends to deflect toward the moving stream. Because of the difficulty in measuring displacement or velocity of the response of the structure, the acceleration is measured instead.

4b-1. Aluminum Structure The instability in the turbulent boundary layer caused surface waves in the structure along the direction of flow. The surface waves decay with distance at a slower rate than that in the flow. Results show that the spatial scale in the boundary layer flow approximated the boundary layer thickness, while in the structure the surface waves propagated over several boundary layer thicknesses. Laterally, the waves had nearly zero phase shift and were nonconvecting or kinematic. The waves maintained high correlation values with distance. Figure 3 shows an example of the instantaneous space and time response $g(x, t)$ from an array of four longitudinal accelerometers placed $12.5 \mathrm{~cm}$ apart between locations 1 and 4 (fig. 1(b)). The convected disturbances on the surface of the structure are indicated in the figure. ${ }^{4,25,26}$

Tear stoppers are used in aircraft structures to prevent cracks from propagating. A tear stopper is a local thick aluminum strip that is thicker than the skin and is glued or riveted to it. As a result, the discontinuity in the surface becomes a source of noise radiation. A classical example is that of an infinite panel (panel without surface discontinuity) radiating no sound as long as the waves are subsonic. To reduce tear stopper noise, the geometry was changed. The aluminum structure is a plate which was machined into six panels separated by tear stoppers. The tear stoppers gradually increase in thickness and decrease into the next panel without changing the total mass compared with a standard tear stopper. (fig. (4)). The use of time averages for space and time correlation along the direction of flow $R\left(x_{1}, x_{2} ; \tau\right)$ where $\tau$ is the time delay (fig. (4)) maintains larger correlations along the length than that of real time snapshots (fig. (3)). The time averages are used to describe a special property of structural waves in the presence of a boundary shown in figure 4. Reduction of waves transmitted through tear stoppers are clearly shown. The boundary provides high attenuation of the transmitted wave. The change in response is attributed to the gradual change in stiffness near the boundary. Note that amplitude and spatial scale reduction occurs when the convected wavelengths are within the tear stopper geometrical scale. Waves with larger spatial scale require a wider tear stopper.

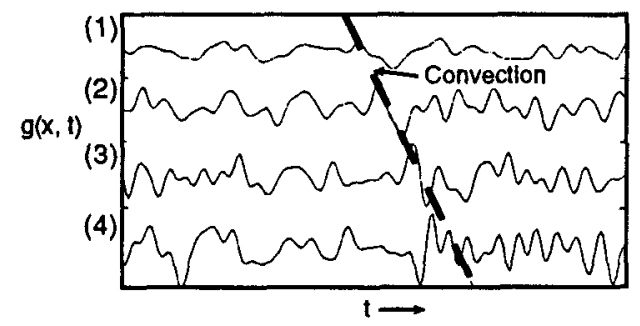

Fig. 3. Aluminum structure, real time response of the spatial distribution, forced by the turbulent boundary layer.

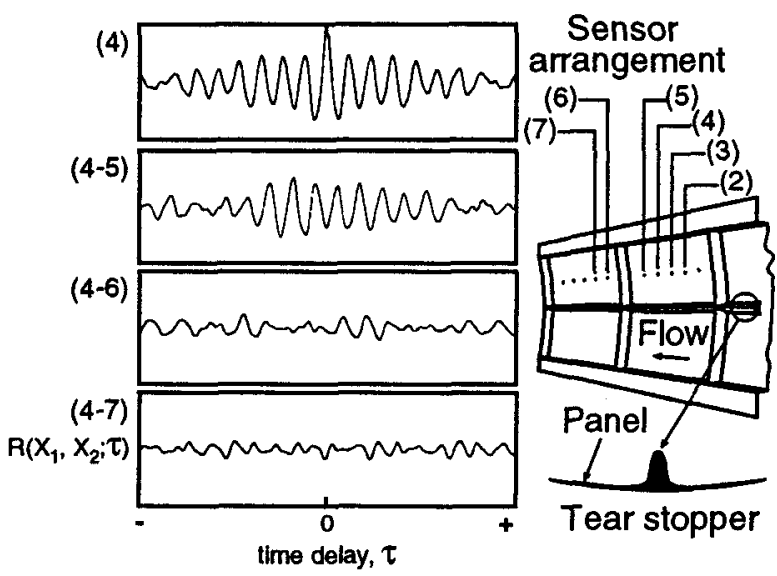

Fig. 4. Aluminum structure, space-time correlation between and across a tear stopper forced by the turbulent boundary layer

Figures 5(a), (b), (c), (d) show the structural response at location 3 forced by four inputs

(a) turbulent boundary layer

(b) turbulent boundary layer with added pure tone sound at $1225 \mathrm{~Hz}$ and $134 \mathrm{~dB}$

(c) turbulent boundary layer, pure tone sound, and sound from $\mathrm{Me}=0.90$ jet

(d) turbulent boundary layer with sound from an underexpanded supersonic jet

The time history $g(t)$, shown for an interval of $0.04 \mathrm{sec}$ near the instant $T$, is used to evaluate the instantaneous spectrum $G(f, T)$, phase $\dot{g}(t)$ versus $g(t)$, and probability $\bar{Q}(r, t)$. Figures $5(\mathrm{a})$, (b) show distinct differences between the response from the turbulent boundary layer and with added pure tone sound. The real time response is amplified, the pure tone frequency with its harmonics and subharmonic exceeds the 
(d)

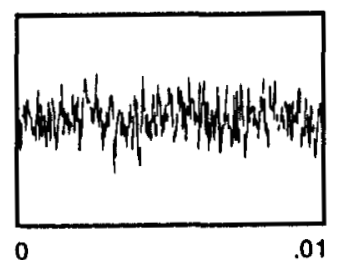

(c)

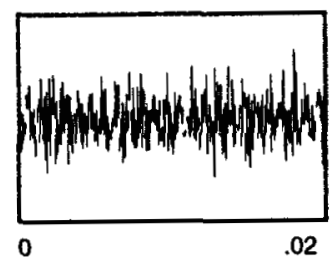

(b)

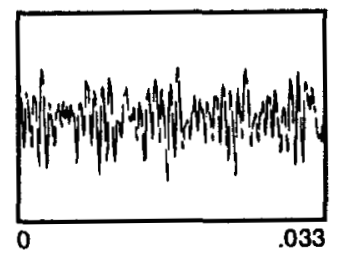

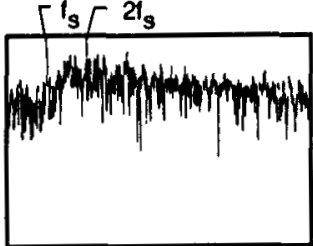
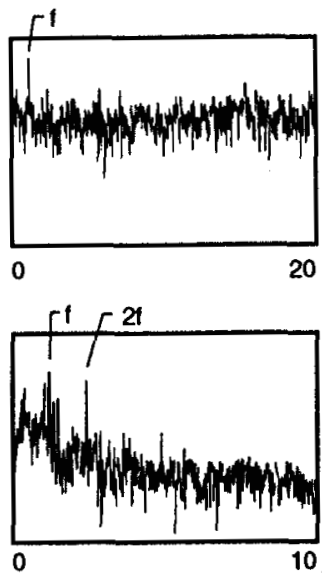
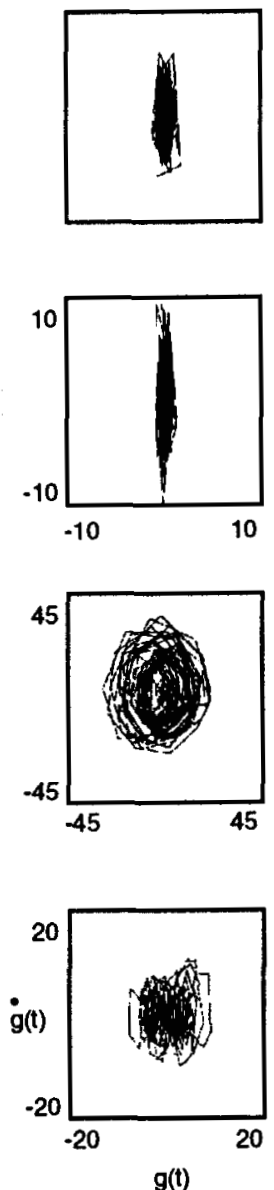
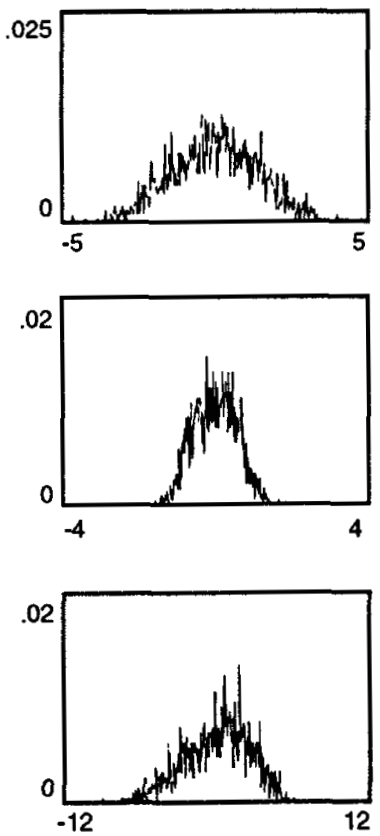
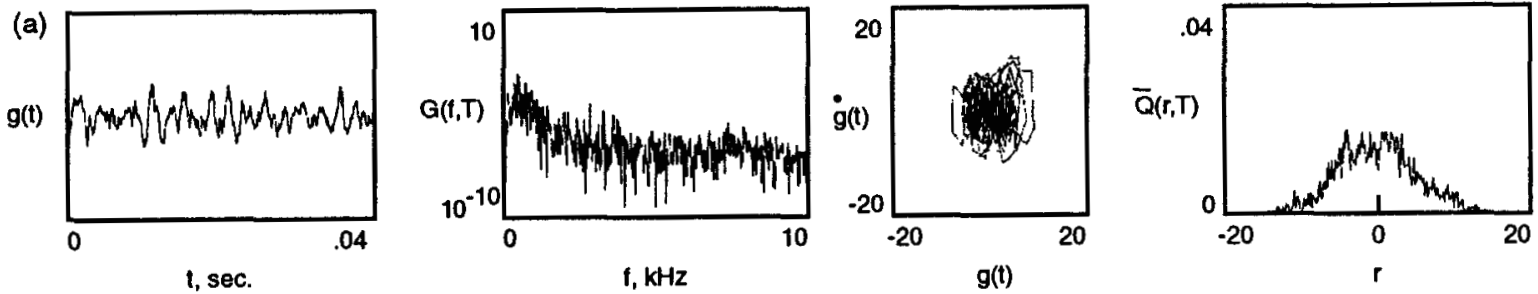

Fig. 5. Aluminum structure, real time response, location (3).

response to the turbulent boundary layer alone. Note the presence of other peaks not harmonically related. The phase plots show the difference in the wall pressure as the pure tone sound is added.

Figure 5(c) shows four plots of the interaction of the pure tone sound with sound from $\mathrm{Me}=0.90$ jet and the structure. The amplitude rises in the broadband spectrum when compared with tone case (fig. 5(b)). The time and phase data have become irregular and the entire record appears nonperiodic, chaotic, and convecting. The fundamental tone maintains a level above the broadband and the probability has a nonsymmetrical distribution. To illustrate the spatial response of the loading for the interaction of the pure tone sound with sound from $\mathrm{Me}=0.90$ jet, four real time plots simultaneously recorded at locations $7,8,9$, and 10 are shown in figure $1(\mathrm{~b})$. The results are shown in figure 6 . The response data indicate the existence of a complicated independent spatial pattern or multiple domains. These figures also indicate that short time scales are highly inhomogeneous, thus providing an additional example of extended uncorrelated space.
Figure 5(d) shows the response of the structure forced by the turbulent boundary layer and sound from an underexpanded supersonic jet at location (3). An increase in amplitude and bandwidth response is noticeable in the presence of screech modes, which are a fundamental and harmonic superimposed on broadband. The result is consistent with the wall pressure fluctuation load input (figs. 2(c), (d)). The phase portraits indicate convective effects, and the probability plots are nearly Gaussian.

Measurements are also made upstream of the nozzle at location 1 where the fundamental screech tone attains a maximum amplitude, and downstream at location 9 where the first harmonic screech tone attains a maximum (fig. 1(b)). Results are shown in figures 7 at locations 1 and 9. The shape and the level of the power spectra and the phase with time plot provide another useful characterization of the convective chaotic fluctuations. The spectrum level is nearly constant and the shock peak frequencies are two orders of magnitude above the broadband level. Extensive measurements have been made in the study of screech 


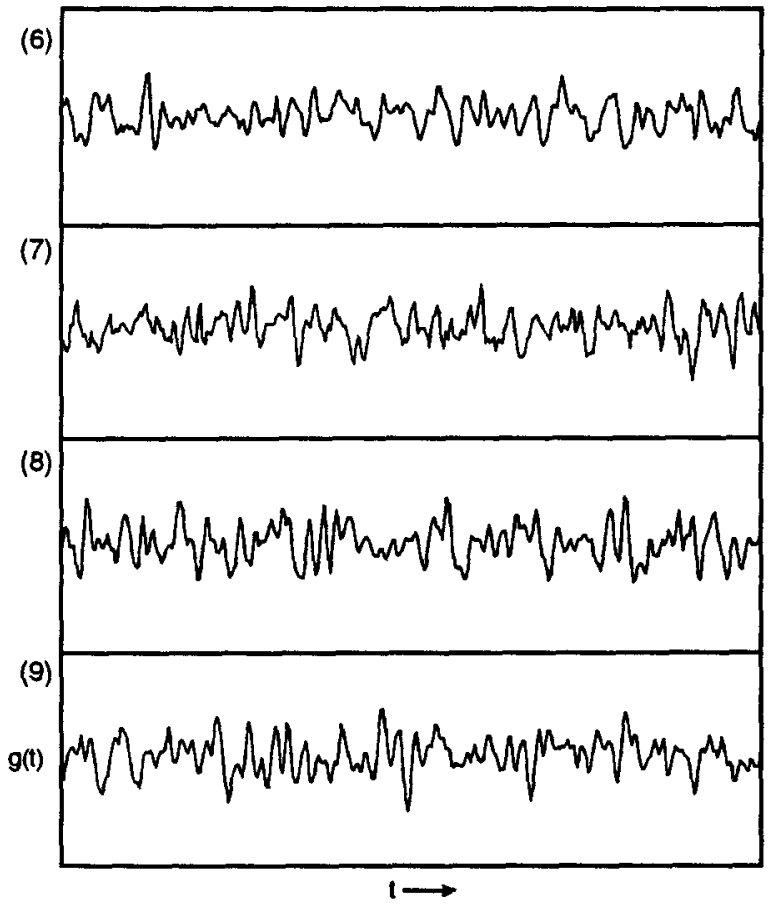

Fig. 6. Aluminum structure, real time response, location (6 to 9 ) forced by turbulent boundary layer with sound from $\mathrm{Me}=0.90$ jet.

in jets. $21,23,27$ It was found that the fundamental tone radiates downstream of the jet axis, and not upstream as indicated in present experiments with a jet exhausting in proximity of a surface.

To substantiate a chaotic state, the Liapunov exponent, $\lambda$ is estimated from data on the structure between locations 1 and 10 (fig. 1(b)). Turbulent boundary layer loads indicate values between -0.3 to -0.02 , while turbulent boundary layer and sound from underexpanded supersonic jet loads indicate values between 0.9 to 1.7. The calculation procedure is repeated until a fiducial estimate is obtained. These numbers quantify the degrees of stability at some point on the surface. The variation of the estimated values indicate the deficiency of the estimation process when using the formula for $\lambda(E, x, y)$. Conditions in which the Liapunov exponent estimate was positive corresponded to conditions in which the standard deviation is nonzero.

$4 b-2$ Graphite-epoxy Structure The graphite-epoxy structure is a single curved sheet without tear stoppers. The response is measured along the center line at locations 3 and 7 (fig. 1(c)). The power spectral density of the acceleration response $G(f, t)$ is shown in figures $8(a),(b)$ without and with pure tone sound forcing on a turbulent boundary layer. The power spectral density is smooth and continuous when excited by the turbulent boundary layer, and the amplitude level is higher than the level observed for the aluminum structure by a factor of four. With the added pure-tone sound (fig. 8 (b)) shows a spike at the forcing frequency of $1225 \mathrm{~Hz}$, which exceeded the amplitude of the broadband level by $25 \mathrm{~dB}$ and the spikes of two harmonics above the broadband. The energy transfer from the fundamental to the harmonics indicates that the forcing amplitude exceeds the threshold value. Note in figure $5(\mathrm{~b})$ that the aluminum structural responses have one harmonic above the broadband, while the graphite-epoxy structure has two harmonics. This difference implies that the threshold value for the aluminum is higher than that of the graphite-epoxy structure. The peak power spectral density level is higher than the aluminum structure by nearly one order of magnitude. At location (7), the harmonics have higher levels with a lower broadband level than location (3). The broadband level at (7) is lower than at (3). This exchange of energy between the broadband and harmonics is due to nonlinear coupling between the turbulent boundary layer and pure tone sound.
(9)

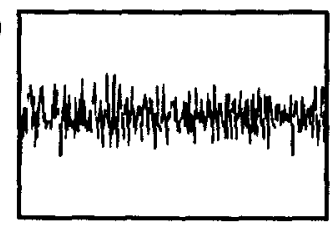

(1)

$\mathbf{g}(\mathbf{t})$

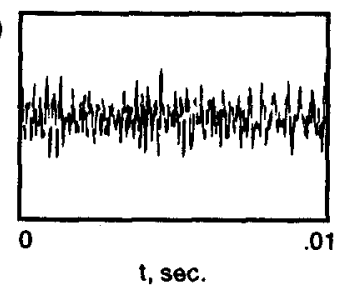

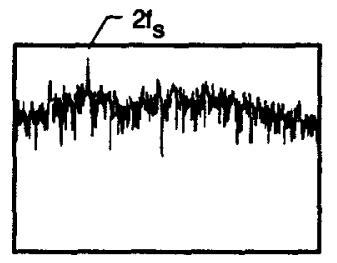

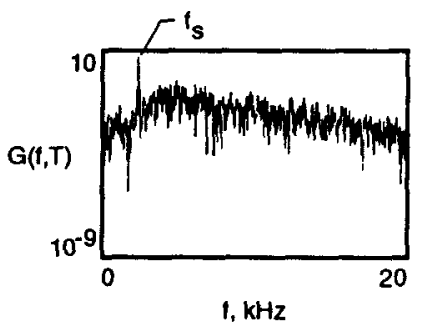

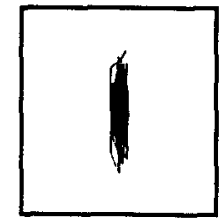

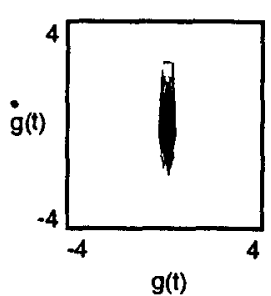

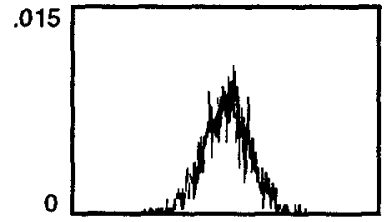

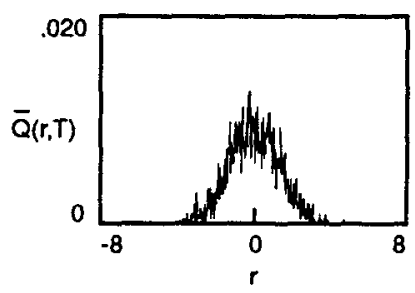

Fig. 7. Aluminum structure, real time response, locations (1) and (9) forced by the turbulent boundary layer and sound from underexpanded supersonic jet. 

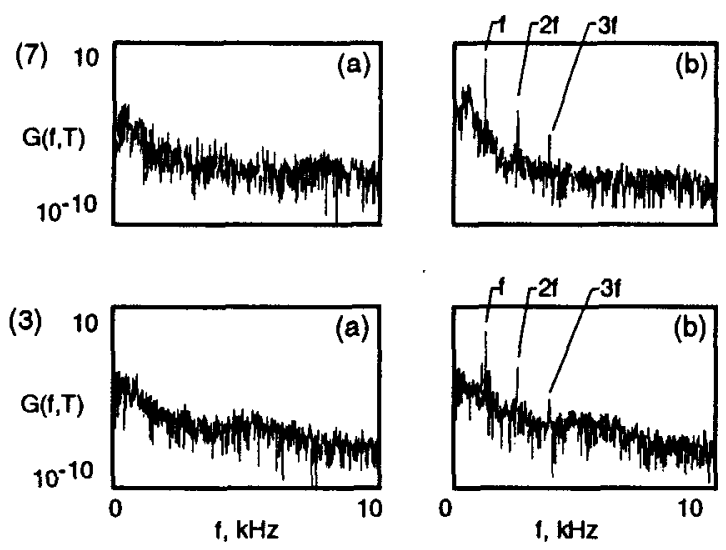

Fig. 8. Graphite-epoxy structure, real time response, location (3) and (7), forced by: (a) turbulent boundary layer, (b) turbulent boundary layer and pure tone sound, $\mathrm{f}=1225 \mathrm{~Hz}$.

When the structure is forced by the sound from an underexpanded supersonic jet on a turbulent boundary layer, the screech tones dominate the broadband response (figs. 9 at location (3) and (7)). A short time history indicates random amplitude, a typical temporal chaotic response behavior, convective phase portrait, and nearly Gaussian distribution. Note that the amplitude of the shock modes increases nearly ten fold above the level of the shock harmonics in the aluminum structure. At location 3 the fundamental dominates the broadband level, while at location 7 the harmonic dominates the broadband level with some contribution from the fundamental.

\section{Sound Transmitted Through Structure}

The turbulent boundary layer with superimposed pure tone sound or sound from the underexpanded supersonic jet induces structural vibration, which in turn induces acoustic pressure in the ambient medium outside the tunnel, simulating the cabin noise. A pressure transducer is placed at $1.6 \mathrm{~m}$ from the structure and near its mid-section. Figures 10(a), (b), (c) and 11(a), (b), (c) show results of the transmitted pressure for aluminum and graphite-epoxy structures respectively. The figures include real time response $p(t)$, power spectral density $P(f, t)$, phase $\dot{p}(t)$ vs $p(t)$, and probability density distributions $\hat{Q}(r, t)$. The transmitted pressure waves are qualitatively similar to the waves of the structure, as well as those input to the structure (figs. 7, 9). The power spectrum density is chaotic and the phase is not periodic. No appreciable difference exists in the level of the transmitted pressure between the two structures, while the vibration level is found to be nearly an order of magnitude higher in the graphite-epoxy than the aluminum structure. The results may not be of great surprise since the construction is different for the two structures. Note that sound radiation comes from the uncancelled volume velocity at the boundary as well from $\mathrm{N}$-wave loads. The graphite-epoxy structure has fewer boundaries and larger responses while the aluminum structure has a greater number of boundaries.

\section{Control of Structure Response}

The pure tone harmonics and $\mathrm{N}$-wave response of the graphite-epoxy structure upstream of the nozzle is stabilized and suppressed by a local external fixed temporal oscillator system. Pioneering work by Ott, Grebogi, York and others has indicated that the time needed to achieve control depends on the transient lifetime between periodic and chaotic cycles. ${ }^{13,33,34}$ It
(7)

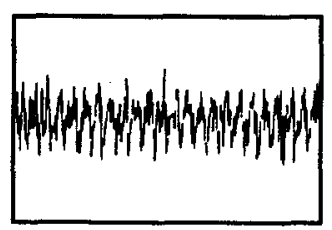

(3)

$g(t)$

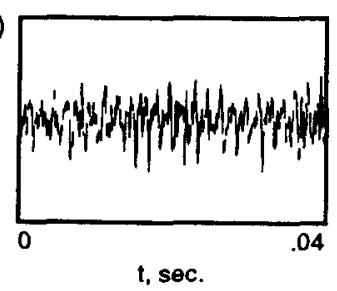

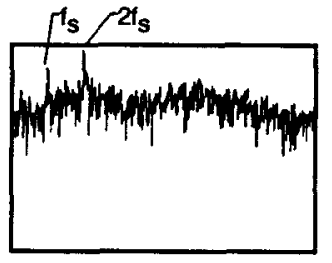
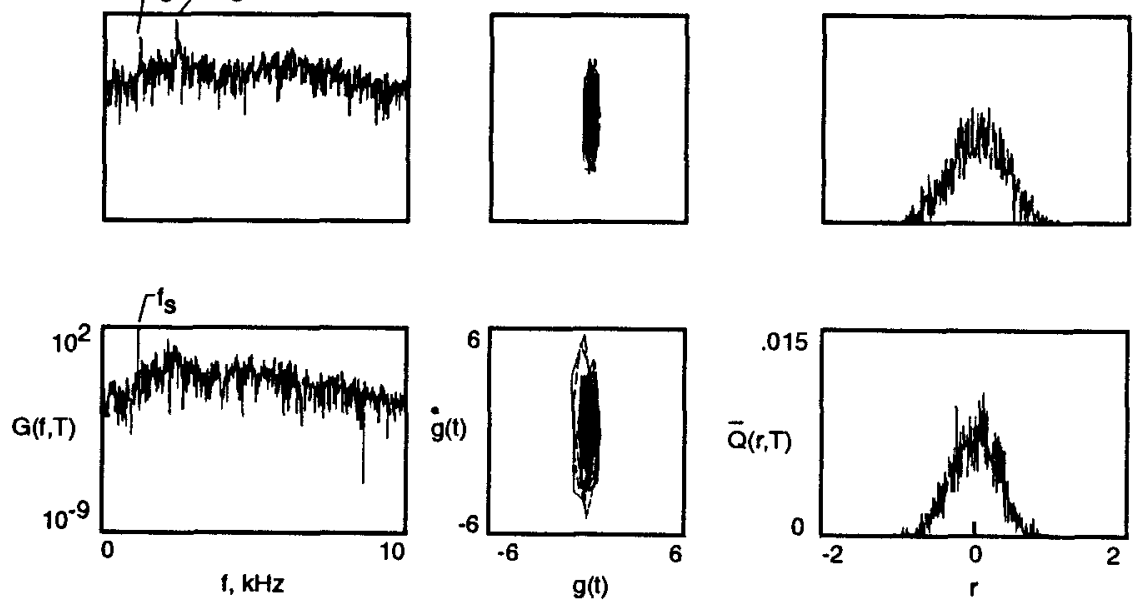

Fig. 9. Graphite-epoxy structure, real time response, location (3) and (7), forced by turbulent boundary layer and sound from underexpanded supersonic jet. 
is necessary to localize the periodic orbit on the surface of the structure, as was done in earlier experiments. 11,20 The dynamics of the structure can be changed by using a small feedback control on the surface. This method allows the elimination of the perturbation of the fundamental and harmonics from pure tone and the $\mathrm{N}$ wave load.

The controller, an electromagnetic actuator, is mounted on the graphite-epoxy structure at location 1 (fig. 1(c)). Two accelerometer signals are notch-filtered around the fundamental tone frequency or around the shock wave fundamental frequency. One accelerometer is placed near location 1 (origin 0,0 ). The other accelerometer is placed at one-quarter length downstream near position 2 and provides the output signal from the panel motion $Z_{i}(t)=g(0,0, t-n)$, where $n(t)$ denotes the time delay or phase shift. The difference $D(t)$ between input and output is used as a control signal

$$
f(t)=K(t)\left[Z_{i}(t)-Z(t)\right]=K(t) D(t)
$$

where $K(t)$ is an adjustable amplitude. Also, the time shift $n(t)$ of $Z_{i}(t)$ with respect to $Z(t)$ can be controlled. The control is introduced into the system input as a negative feedback $(K>0)$. When the control is accomplished, the output signal $Z(t)$ is close to $Z_{i}(t)$, and hence the controlling force $F(t)$ becomes small. At least two and up to four control level adjustments of $K$ and the phase lag $n$ are made by the feedback system.
The response is controlled by forcing the structure at the fundamental frequency through a phase and amplitude tuning stages. In the first stage, the controller forces the panel with amplitude and phase variations $K(t)$ and $n(t)$ so that the energy in the harmonics is shifted back into the fundamental tone. In the second stage, the controller reduces the amplitude of the fundamental by again changing the amplitude and phase. Control is not straightforward, and full control is not always achieved. By comparing the plots in figures 12(c) and 13(c) with those in figures 12(b), and 13(b) and 12(a), and 13(a), the remarkable changes in the level of the pure tone and harmonics is seen, as well as in the level of the shock fundamental mode. The peak amplitude level of the structure is reduced about two orders of magnitude from that without control to nearly the level without external acoustic loadings. Similar changes occur in time plots, phase, and probability. Thus, the system dynamics can effectively be controlled with a small applied force via a single controller.

\section{Conclusion}

Nonlinear response of two structures when forced by turbulent boundary layer with high intensity tonal sound or with sound from an underexpanded supersonic jet in forward motion were experimentally determined. The structures were curved; one was made of aluminum with tear stoppers and the other was made of graphiteepoxy without tear stoppers. (c)

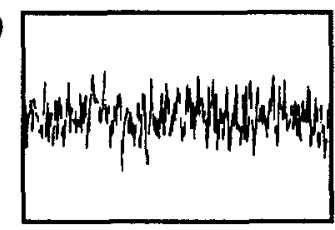

(b)

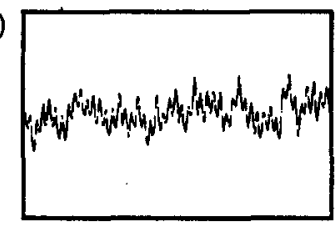

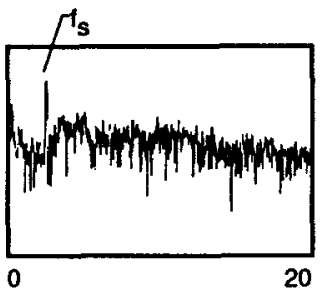
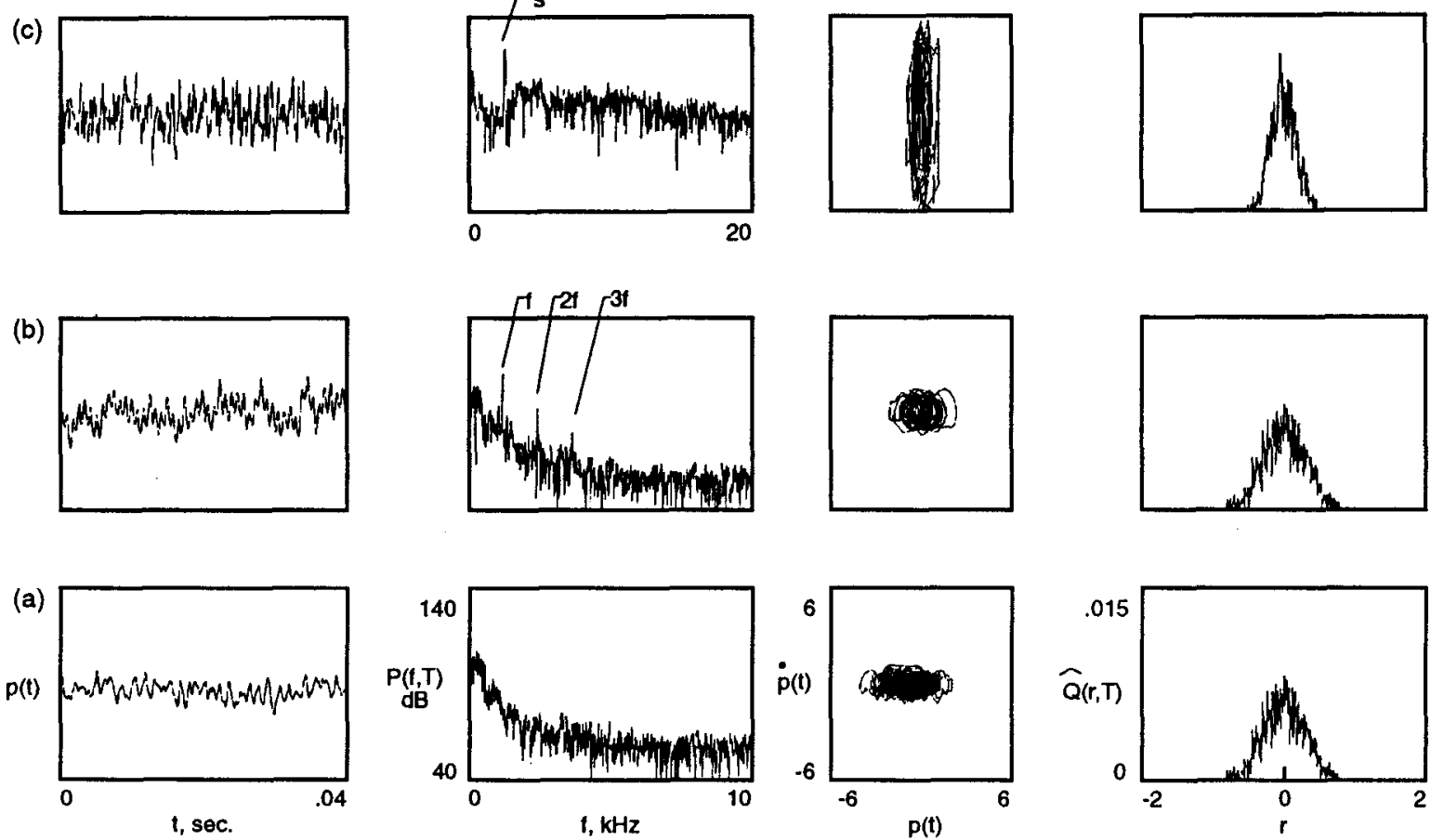

Fig. 10. Aluminum structure, transmitted pressure, (a) turbulent boundary layer, (b) turbulent boundary layer and pure tone sound $\mathrm{f}=1225 \mathrm{~Hz}$, (c) turbulent boundary layer and sound from underexpanded supersonic jet. 
(c)

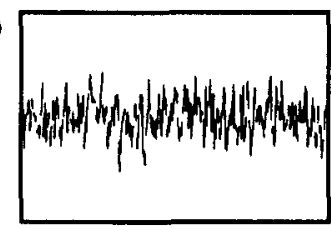

(b)

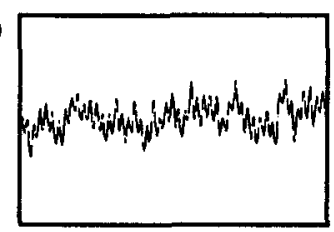

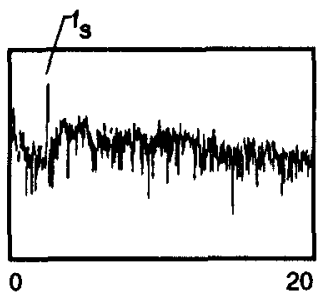
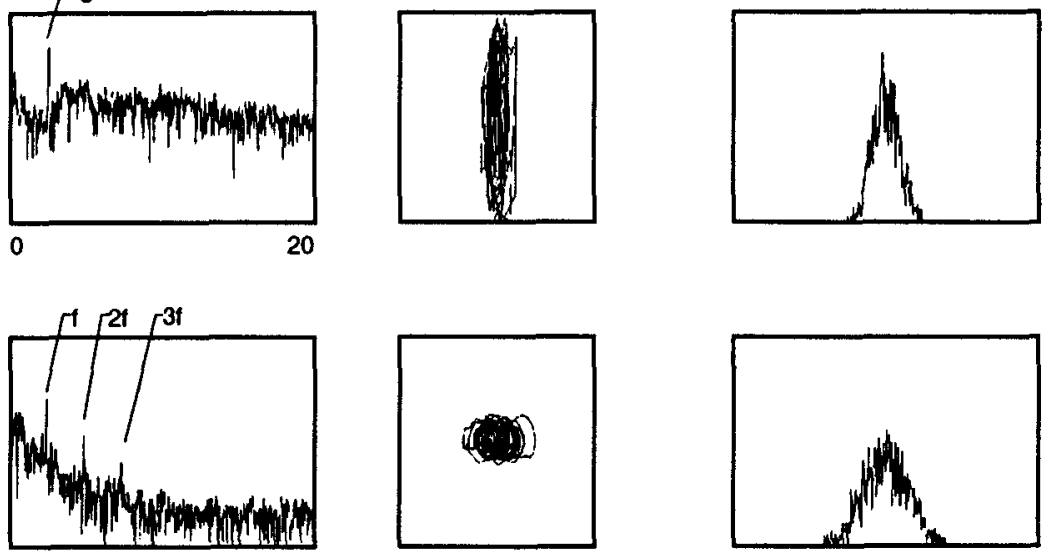

(a)
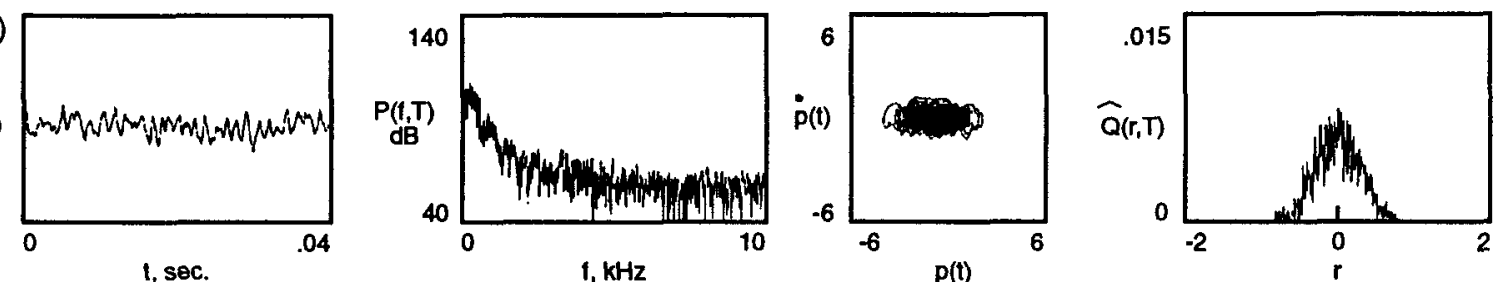

Fig. 11. Graphite-epoxy structure, transmitted pressure, (a) turbulent boundary layer, (b) turbulent boundary layer and pure tone sound $\mathrm{f}=1225 \mathrm{~Hz}$, turbulent boundary layer and sound from underexpanded supersonic jet.

(c)

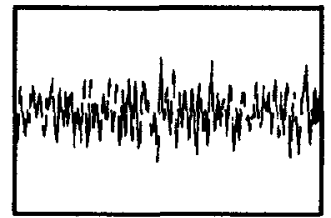

(b)

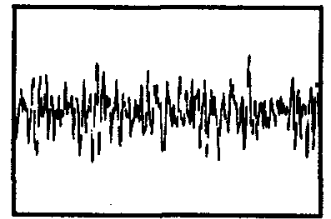

(a)

$g(t)$

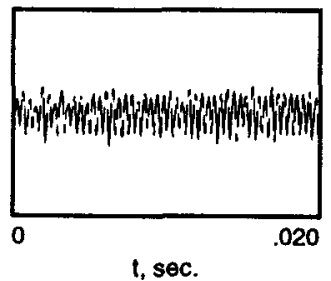

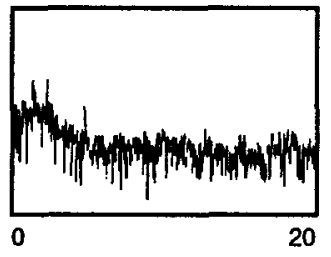
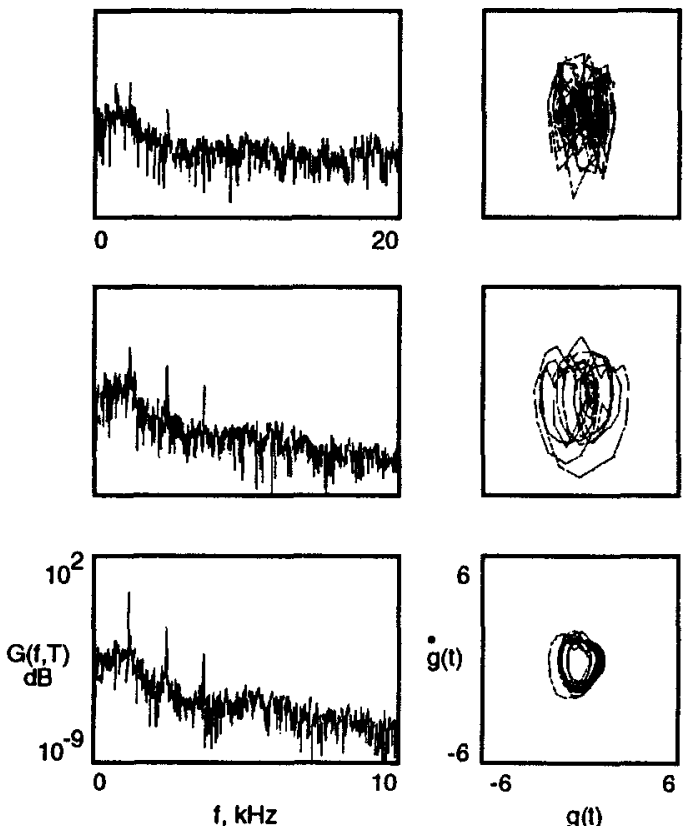
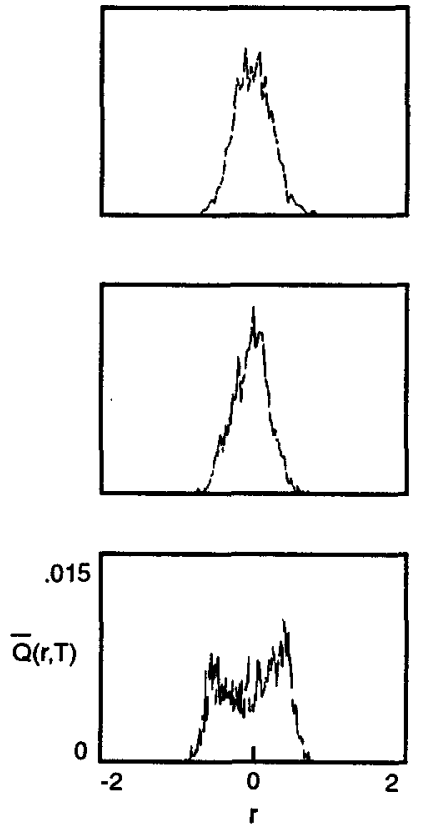

Fig. 12. Graphite-epoxy structure, active control of the acceleration response forced by turbulent boundary layer and pure tone sound $\mathrm{f}=1225 \mathrm{~Hz}$, location (1): (a) uncontrol, (b) partial control, (c) total control. 
(c)

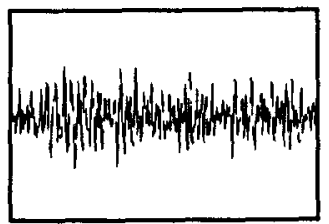

(b)
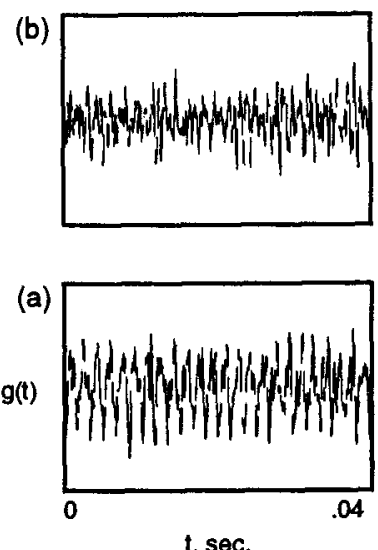
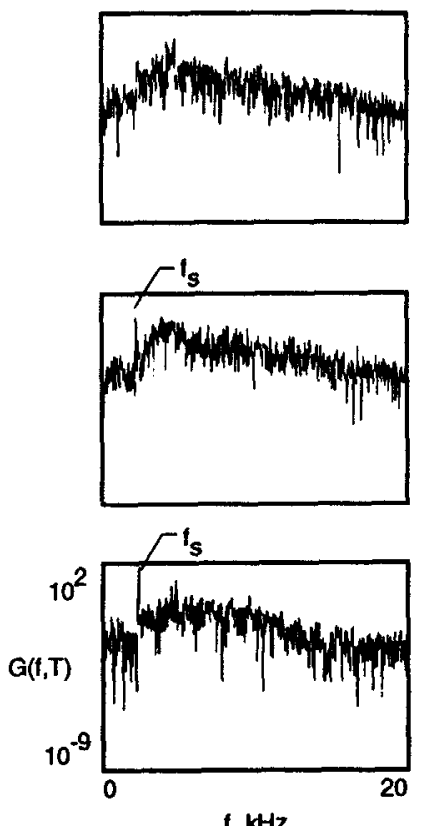
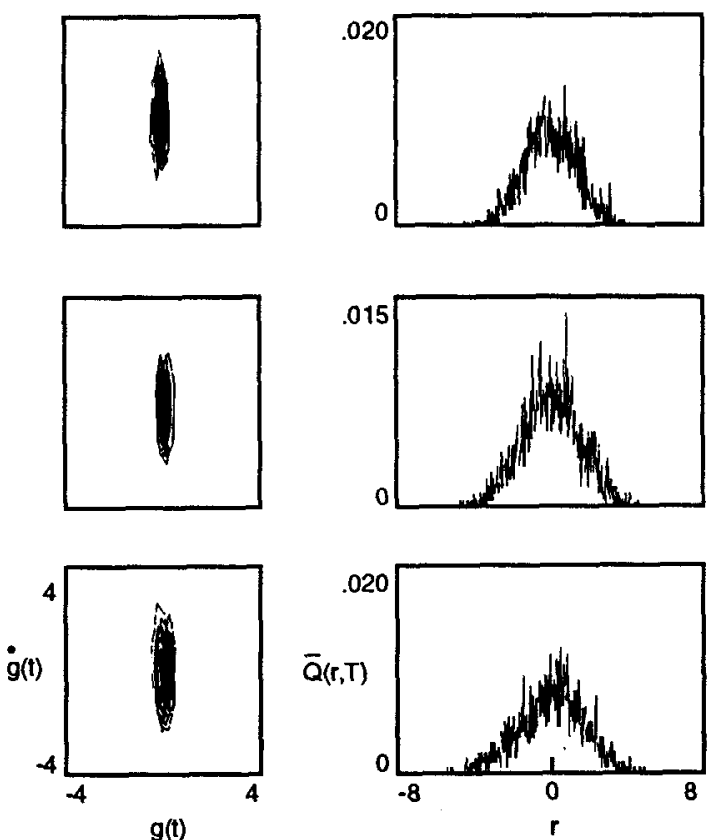

Fig. 13. Graphite-epoxy structure, active control of the acceleration response forced by turbulent boundary layer and sound from underexpanded supersonic jet, location (1): (a) uncontrolled, (b) partial control, (c) total control.

From these results, the following is summarized:

1. The wall pressure fluctuation with an impinging shock manifests different spatial behaviors due to the different modes and their angular components.

2. The structure when loaded by both turbulent boundary layer and sound produces a random generation of patterns.

3. Multiple real time observation points are essential.

4. Waves with wavelengths shorter than the tear stopper are attenuated. A longer wavelength requires a wider tear stopper.

5. By using an external oscillator, tonal sound, and shock mode components on a structure can be stabilized.

6. The response of the graphite-epoxy structure exceeds the response of the aluminum structure, while the transmitted pressures have nearly equal levels. This indicates that tear stoppers are a source of sound.

7. By actively controlling the response of the graphite-epoxy structure, the level is reduced to nearly that of the aluminum structure.

The problem of spatiotemporal response of nonlinear structural dynamics deserves further research. A concern is maintaining the reality of physical behavior with the constraint imposed by the unsteady changes in spatial domains. The approach used and the results described are still in the early stages of development. Data analysis and control methods are based more or less on intuition or experience rather than rigorous arguments.

\section{References}

1. Bayliss, A., and Maestrello, L., "Nonaxisymmetric Disturbances in a Jet and Their Interaction with a Nearby Array of Fuselage Panels." Fourth AIAA/CEAS Joint Aeroacoustics Conference, June 2-4, 1998, Toulouse, France.

2. Chow, P.L., and Maestrello, L., "Active Control of Nonlinear Panel Vibration and Sound Radiation," Journal of Sound and Vibration, 201(3), 1997, pp. 390-403.

3. Fenno, C.C.; Bayliss, A.; and Maestrello, L.: Panel Structure Response to Acoustic Forcing by a Nearby Sonic Jet. AIAA Journal, Vol. 35, 1997, pp. 219-227.

4. Landau, L., and Lifshitz, E.M., "Mechanics of Continuous Media," Fitmatigiz, Moscow (in Russian), 1954.

5. Soderstrom, T , and Stoica, P. "System Identification," Prentice Hall, London, 1989.

6. Cross, M.C. and Hohenberg, P.C., "Pattern Formation Outside of Equilibrium," Rev. Mod. Phys., Vol. 65, No. 3, July 1993, pp. 865-97.

7. Ruelle, D., and Takens, F., "On the Nature of Turbulence," Communication Mathematics Physics, 20, pp. 167-192, 255, 315,316, 538.

8. Dowell, E.H., "Flutter of a Buckled Plate as an Example of Chaotic Motion of a Deterministic Autonomous System," Journal of Sound and Vibration 85(3) 1982, pp. 333-344. 
9. Dowell, E.H., "Nonlinear Oscillations of a Fluttering Plate, Part I and II," AIAA Journal 4, 1996, pp. 1267-1275.

10. Nayfeh, A.H., Rouf, R.A., and Nayfeh, J. F., and Mook, D.T., "Nonlinear Response of Infinitely Long Cylindrical Shells," J. of Applied Mechanics 54, 1987, pp. 571-577.

11. Maestrello, L., "Active Control of Panel Oscillation Induced by Accelerating Boundary Layer and Sound," AIAA Journal, Vol. 35, No. 5, May 1997.

12. Maestrello, L., "Spatiotemporal Chaos of Forced Panel Vibrations, Induced by Turbulent Boundary Layer and Sound," AIAA Journal, to be published.

13. Ott, E., Grebogi, C., and Yorke, J.A., "Controlling Chaos," Phys. Rev. Letters, Vol. 64, No. 1, 1990, pp. 1196-1199.

14. Gollup, J.P. and Ramshanker, R., "Spatiotemporal Chaos in Interfacial Waves," New Perspectives in Turbulence, edited by Sirovich, Chapter 6, Springer-Verlag, 1991, pp. 165-194.

15. Ruelle, D., "The Glaude Bernard Lecture, 1989: Deterministic Chaos, The Science and the Fiction," Proc R. Soc. London. A, 1990, pp. 261-268.

16. Sahay. A., and Sreenivasan, K.R., "The Search for a Low-Dimensional Characterization of a Local Climate System." Phil. Trans. R. Soc. London. A, 1996, 354, pp. 1715-1750.

17. Eckmann, J.P. and Ruelle, D., "Ergotic Theory of Chaos and Strange Attractors," Rev., Mod. Phys. 57, 1985, pp. 617-656.

18. Screenivasan, K.R.," Transition and Turbulence in Fluid Flow and Low-Dimensional Chaos," Frontia in Fluid Machanics, edited by S.H. Davis and J.M. Lumley, Springer- Verlag, 1985, pp. 41-67.

19. Hurre, P., and Monkewitz, P.A., "Local and Global Instability in Spatially Developing Flow." Annual Rev. of Fluid Mech. Vol. 22 22, 1990, pp. 473-537.

20. Maestrello, L., "Control of Nonlinear-Nonstationary Structural Response and Radiation Near a Supersonic Jet," AIAA Journal, Vol. 32, No. 7, July 1994.

21. Powell, A.," On the Mechanism of Choked Jet Noise," Proc. Phys. Soc. 66, Vol. PE12, No. 408B, London, 1953, pp. 1039-1056.

22. Norum, T. D. "Screech Suppression in Supersonic Jets," AIAA Journal, Vol. 21, No. 2, Feb. 1983. pp. 235-240.
23. Norum, T.D., and Seiner, J.M., "Location and Propagation of Shock Associated Noise from Supersonic Jet," AIAA Journal, Vol. 20, Jan. 1982, pp. 68-73.

24. Fenno Jr., C.C., Bayliss, A., and Maestrello, L., "Panel-Structure Response to Acoustic Forcing by a Nearly Sonic Jet," AIAA Journal, Vol. 35, No. 2, 1997, pp. 219-227.

25. Maestrello, L., "Use of Turbulent Model to Calculate the Vibration and Radiation Responses of a Panel with Practical Suggestion of Resolving Sound Level," Journal Sound and Vibration, 1969, pp. 405-448.

26. Baht, W.V. and Wilby, J.F., "Interior Noise Radiated by an Airplane Fuselage Subjected to Turbulent Boundary Layer Excitation and Evaluation of Noise Reduction Treatments," Journal of Sound and Vibration, 18(4), 1971, pp. $449-464$.

27. Hilborn, R.C., "Chaos and Nonlinear Dynamics," Chapter 4, Oxford University Press, Inc., 1994.

28. Chaté, H. and Manneville, P., "Spatiotemporal Intermittency," in: A Tentative Dictionary of Turbulence, Tabeling, P. and Cardoso, O. eds., Plenum, New York, 1995.

29. Principe, L ., and Rathie, A. and Kuo, J. M., "Prediction of Chaotic Time Series with Neural Networks and Issue of Dynamic Modeling," Int. Journal Bifurcation and Chaos 2(4), 1992, pp. 989-996.

30. Maestrello, L., "Control of Panel response to Turbulent Boundary Layer and Acoustic Excitation," AIAA, Vol. 24, No. 2, 1996, pp. 259-264

31. Grassberg, P. and Procaccia, I., "Characterization of Strange Attractors," Physical Review Letters, Vol. 50, N. 5, 1983, pp. 346-349.

32. Grassberger, P., and Procaccia, I., "Measuring the Strangeness of Strange Attractors," Physica, D9, 1983b, pp. 189.

33. Tamas Tel, "Controlling Transient Chaos," J. Phys. A: Math. Gen. 24,1991, L1359-L1368.

34. Chen, G. and Dong, X., "From Chaos to OrdersPerspectives and Methodologies in Controlling Chaotic Nonlinear Dynamical System," International Journal of Bifurcation and Chaos, Vol. 3, 1993, pp. 1363-1409. 